Pacific

Journal of

Mathematics

\title{
WITTEN GENUS AND STRING COMPLETE INTERSECTIONS
}

Qingtao Chen And Fei HaN

Volume $234 \quad$ No. 2

February 2008 


\title{
WITTEN GENUS AND STRING COMPLETE INTERSECTIONS
}

\author{
Qingtao Chen And Fei Han
}

\begin{abstract}
We prove the vanishing of the Witten genus of generalized string complete intersections in products of complex projective spaces. Our result generalizes a known result of Landweber and Stong.
\end{abstract}

\section{Introduction}

Let $M$ be a $4 k$-dimensional closed oriented smooth manifold. Let $E$ be a complex vector bundle over $M$. For any complex number $t$, set

$$
\begin{aligned}
\Lambda_{t}(E) & =\mathbb{C} \mid M+t E+t^{2} \Lambda^{2}(E)+\cdots, \\
S_{t}(E) & =\mathbb{C} \mid M+t E+t^{2} S^{2}(E)+\cdots,
\end{aligned}
$$

where for any integer $j \geq 1, \Lambda^{j}(E)$ is the $j$-th exterior power of $E$ and $S^{j}(E)$ is the $j$-th symmetric power of $E$; see [Atiyah 1967]. Set $\widetilde{E}=E-\mathbb{C}^{\mathrm{rk}(E)}$.

Let $q=e^{\pi i \tau}$ with $\tau \in \mathbb{H}$, the upper half plane. Witten [1988] defined

$$
\Theta_{q}(E)=\bigotimes_{n \geq 1} S_{q^{2 n}}(E)
$$

and then defined $\varphi_{W}(M)$, now called the Witten genus, for $M$ as

$$
\varphi_{W}(M)=\left\langle\widehat{A}(M) \operatorname{ch}\left(\Theta_{q}(\widetilde{T M \otimes \mathbb{C}})\right),[M]\right\rangle,
$$

where $\widehat{A}(M)$ is the $\widehat{A}$-characteristic class of $M$ and $[M]$ is the fundamental class of $M$. (See [Zhang 2001] for definitions of $\widehat{A}$ and ch by curvature in Chern-Weil theory.) Let $\left\{ \pm 2 \pi \sqrt{-1} x_{j}\right.$ for $\left.1 \leq j \leq 2 k\right\}$ be the formal Chern roots of $T M \otimes \mathbb{C}$. From [Liu 1996; 1995b], the Witten genus can be written using Chern roots as

$$
\varphi_{W}(M)=\left\langle\prod_{j=1}^{2 k} x_{j} \frac{\theta^{\prime}(0, \tau)}{\theta\left(x_{j}, \tau\right)},[M]\right\rangle,
$$

where $\theta(v, \tau)$ is the Jacobi theta function; see page 252 below. The Witten genus, which he introduced for studying two-dimensional supersymmetric quantum field theory, can be viewed as the loop space analogue of the $\widehat{A}$ genus.

MSC2000: primary 57R20, 53C20; secondary 11Z05, 53C80.

Keywords: Witten genus. 
By the Atiyah-Singer index theorem, when $M$ is spin, $\varphi_{W}(M) \in \mathbb{Z} \llbracket q \rrbracket$; see [Hirzebruch et al. 1992]. Moreover, if the spin manifold $M$ is string, that is, $p_{1}(T M) / 2=0-p_{1}(T M) / 2$ is a cohomology class in $H^{4}(M, \mathbb{Z})$, twice of which is the first integral Pontryagin class $p_{1}(T M)$ - or even weaker, if the first rational Pontryagin class $p_{1}(M)=0$, then $\varphi_{W}(M)$ is a modular form of weight $2 k$ with a $q$-series expansion of integer coefficients; see [Hirzebruch et al. 1992]. (For a study of the Witten genus of $\operatorname{spin}^{\mathrm{c}}$ manifolds, see [Zhang 1992].)

Let $V_{\left(d_{p q}\right)}$ be a nonsingular $4 k$-dimensional generalized complete intersection in the product of complex projective spaces $\mathbb{C P}^{n_{1}} \times \mathbb{C P}^{n_{2}} \times \cdots \times \mathbb{C} \mathrm{P}^{n_{s}}$, which is dual to $\prod_{p=1}^{t}\left(\sum_{q=1}^{s} d_{p q} x_{q}\right) \in H^{2 t}\left(\mathbb{C P}^{n_{1}} \times \mathbb{C P}^{n_{2}} \times \cdots \times \mathbb{C P}^{n_{s}}, \mathbb{Z}\right)$, where $x_{q} \in H^{2}\left(\mathbb{C P}^{n_{q}}, \mathbb{Z}\right)$ for $1 \leq q \leq s$ is the generator of $H^{*}\left(\mathbb{C P}^{n_{q}}, \mathbb{Z}\right)$ and $d_{p q}$ for $1 \leq p \leq t$ and $1 \leq q \leq s$ are integers. Let $P_{q}: \mathbb{C P}^{n_{1}} \times \cdots \times \mathbb{C P}^{n_{s}} \rightarrow \mathbb{C P}^{n_{q}}$ for $1 \leq q \leq s$ be the $q$-th projection. $V_{\left(d_{p q}\right)}$ is the intersection of the zero loci of smooth global sections of line bundles $\otimes_{q=1}^{s} P_{q}^{*}\left(\mathcal{O}\left(d_{p q}\right)\right)$ for $1 \leq p \leq t$, where $\mathcal{O}\left(d_{p q}\right)=\mathscr{O}(1)^{d_{p q}}$ is the $d_{p q}$-th power of the canonical line bundle $\mathbb{O}(1)$ over $\mathbb{C P}^{n_{q}}$.

Here we have somewhat abused the terminology of complete intersection from algebraic geometry. We don't require that the integers $d_{p q}$ be nonnegative, so $V_{\left(d_{p q}\right)}$ might not be an algebraic variety. However, by transversality, $V_{\left(d_{p q}\right)}$ can always be chosen to be smooth. $V_{\left(d_{p q}\right)}$ is a representative in $\mathbb{C P}^{n_{1}} \times \cdots \times \mathbb{C P}^{n_{s}}$ of the virtual submanifold $\left(\sum_{q=1}^{s} d_{1 q} \chi_{q}, \ldots, \sum_{q=1}^{s} d_{t q} \chi_{q}\right)$, in the sense of [Hirzebruch et al. 1992]. Putting certain conditions (Proposition 3.1 below) on the data $n_{q}$ for $1 \leq q \leq s$ and $d_{p q}$ for $1 \leq p \leq t$ and $1 \leq q \leq s$, the complete intersection $V_{\left(d_{p q}\right)}$ can be made string. When applied systematically, this generates many nice examples of string manifolds, whose Witten genus we study here. See also [Gorbounov and Malikov 2004; Gorbounov and Ochanine 2006] for a study of elliptic genera of complete intersections and the Landau-Ginzburg/Calabi-Yau correspondence.

Set

$$
D=\left[\begin{array}{llll}
d_{11} & d_{12} & \cdots & d_{1 s} \\
d_{21} & d_{22} & \cdots & d_{2 s} \\
\cdots & \cdots & \cdots & \cdots \\
d_{t 1} & d_{t 2} & \cdots & d_{t s}
\end{array}\right] .
$$

and let $m_{q}$ be the number of nonzero elements in the $q$-th column of $D$. The main result of this paper is this:

Theorem 1.1. If $m_{q}+2 \leq n_{q}$ for $1 \leq q \leq s$ and $V_{\left(d_{p q}\right)}$ is string, then the Witten genus $\varphi_{W}\left(V_{\left(d_{p q}\right)}\right)$ vanishes.

Our result generalizes the known vanishing of the Witten genus of string nonsingular complete intersections of hypersurfaces with degrees $d_{1}, \ldots, d_{t}$ in a single complex projective space, a result due to Landweber and Stong which is described by Hirzebruch, Berger, and Jung [1992, Section 6.3] by applying the properties of 
the sigma function. Stolz [1996] used this as evidence of his celebrated conjecture. In the special case we treat, our result is broader than theirs, since we don't require that the $d_{1}, \ldots, d_{t}$ are all positive.

Explicitly expanding $\Theta_{q}(\widehat{T M \otimes \mathbb{C}})$, we get

$$
\widehat{A}(M) \operatorname{ch}\left(\Theta_{q}(\widehat{T M \otimes \mathbb{C}})\right)=\widehat{A}(M)+\widehat{A}(M)(\operatorname{ch}(T M \otimes \mathbb{C})-4 k) q^{2}+\cdots .
$$

Therefore it's not hard to obtain this corollary from Theorem 1.1:

Corollary 1.2. If $m_{q}+2 \leq n_{q}$ for $1 \leq q \leq s$ and $V_{\left(d_{p q}\right)}$ is string, then

$$
\left\langle\widehat{A}\left(V_{\left(d_{p q}\right)}\right),\left[V_{\left(d_{p q}\right)}\right]\right\rangle=0 \text { and }\left\langle\widehat{A}\left(V_{\left(d_{p q}\right)}\right) \operatorname{ch}\left(T V_{\left(d_{p q}\right)} \otimes \mathbb{C}\right),\left[V_{\left(d_{p q}\right)}\right]\right\rangle=0 \text {. }
$$

Let $M$ be a 12-dimensional oriented closed smooth manifold. The signature of $M$ can be expressed by the $\widehat{A}$-genus and the twisted $\widehat{A}$-genus as

$$
\{\hat{L}(M)\}^{(12)}=\left\{8 \widehat{A}(M) \operatorname{ch}\left(T_{\mathbb{C}} M\right)-32 \widehat{A}(M)\right\}^{(12)} ;
$$

see [Alvarez-Gaumé and Witten 1984; Liu 1995a]. See [Zhang 2001] for a definition of $\hat{L}$ by curvature in Chern-Weil theory. This formula and its higher generalization have an application to the Rokhlin-Ochanine divisibility of the signature; see [Ochanine 1981; Liu 1995a]. Combining Corollary 1.2 and (1-3), we obtain:

Corollary 1.3. If $m_{q}+2 \leq n_{q}$ for $1 \leq q \leq s$ and $V_{\left(d_{p q}\right)}$ is 12-dimensional and string, then the signature of $V_{\left(d_{p q}\right)}$ vanishes.

Let $M$ be a 16-dimensional oriented closed smooth manifold. From [Chen and Han 2006] we have the formula

$$
\left\{\hat{L}(M) \operatorname{ch}\left(T_{\mathbb{C}} M\right)\right\}^{(16)}=-2048\left\{\widehat{A}(M) \operatorname{ch}\left(T_{\mathbb{C}} M\right)-48 \widehat{A}(M)\right\}^{(16)} .
$$

Corollary 1.4. If $m_{q}+2 \leq n_{q}, 1 \leq q \leq s, V_{\left(d_{p q}\right)}$ is 16-dimensional and string, then the twisted signature $\operatorname{Sig}\left(V_{\left(d_{p q}\right)}, T\right)$ vanishes.

This is proved by combining Corollary 1.2 and (1-4) for the twisted signature

$$
\operatorname{Sig}(M, T) \triangleq\left\langle\hat{L}(T M) \operatorname{ch}\left(T_{\mathbb{C}} M\right),[M]\right\rangle .
$$

\section{Some preliminaries}

This section reviews some tools and the knowledge that we will apply in Section 3 to prove Theorem 1.1. We start with results on residues in complex geometry. See [Griffiths and Harris 1994, Chapter 5] for details.

Let $U$ be the ball $\left\{z \in \mathbb{C}^{s}:\|z\|<\varepsilon\right\}$, and let $f_{1}, \ldots, f_{s} \in \mathcal{O}(\bar{U})$ be functions holomorphic in a neighborhood of the closure $\bar{U}$ of $U$. We assume that each $f_{i}(z)$ has an isolated common zero at the origin. Set

$$
D_{i}=\left(f_{i}\right)=\text { divisor of } f_{i} \text { and } D=D_{1}+\cdots+D_{s} .
$$


Let

$$
\omega=\frac{g(z) d z_{1} \wedge \cdots \wedge d z_{s}}{f_{1}(z) \cdots f_{s}(z)}
$$

be a meromorphic $s$-form with polar divisor $D$. The residue of $\omega$ at the origin is

$$
\operatorname{Res}_{\{0\}} \omega=\left(\frac{1}{2 \pi \sqrt{-1}}\right)^{s} \int_{\Gamma} \omega
$$

where $\Gamma$ is the real $s$-cycle defined by $\Gamma=\left\{z:\left|f_{i}(z)\right|=\varepsilon\right.$ for $\left.1 \leq i \leq s\right\}$ and oriented by $d\left(\arg f_{1}\right) \wedge \cdots \wedge d\left(\arg f_{s}\right) \geq 0$.

Let $M$ be a compact complex manifold of dimension $s$. Suppose that $D_{i}$ for $i=1, \ldots, s$ are effective divisors, the intersection of which is a finite set of points. Let $D=D_{1}+\cdots+D_{s}$. Let $\omega$ be a meromorphic $s$-form on $M$ with polar divisor $D$. For each point $P \in D_{1} \cap \cdots \cap D_{s}$, we may restrict $\omega$ to a neighborhood of $U_{P}$ of $P$ and define the residue $\operatorname{Res}_{P} \omega$ as above. Then ([Griffiths and Harris 1994, Chaper 5]), one has:

Lemma 2.1 (Residue theorem).

$$
\sum_{P \in D_{1} \cap \cdots \cap D_{s}} \operatorname{Res}_{P} \omega=0
$$

We also need some facts about the Jacobi theta functions. Although we are going to use only one of them, we list for completeness all their definitions and transformation laws.

The four Jacobi theta functions are defined below; see for example [Chandrasekharan 1985].

$$
\begin{aligned}
& \theta(v, \tau)=2 q^{1 / 4} \sin (\pi v) \prod_{j=1}^{\infty}\left[\left(1-q^{2 j}\right)\left(1-e^{2 \pi \sqrt{-1} v} q^{2 j}\right)\left(1-e^{-2 \pi \sqrt{-1} v} q^{2 j}\right)\right], \\
& \theta_{1}(v, \tau)=2 q^{1 / 4} \cos (\pi v) \prod_{j=1}^{\infty}\left[\left(1-q^{2 j}\right)\left(1+e^{2 \pi \sqrt{-1} v} q^{2 j}\right)\left(1+e^{-2 \pi \sqrt{-1} v} q^{2 j}\right)\right], \\
& \theta_{2}(v, \tau)=\prod_{j=1}^{\infty}\left[\left(1-q^{2 j}\right)\left(1-e^{2 \pi \sqrt{-1} v} q^{2 j-1}\right)\left(1-e^{-2 \pi \sqrt{-1} v} q^{2 j-1}\right)\right] \\
& \theta_{3}(v, \tau)=\prod_{j=1}^{\infty}\left[\left(1-q^{2 j}\right)\left(1+e^{2 \pi \sqrt{-1} v} q^{2 j-1}\right)\left(1+e^{-2 \pi \sqrt{-1} v} q^{2 j-1}\right)\right]
\end{aligned}
$$

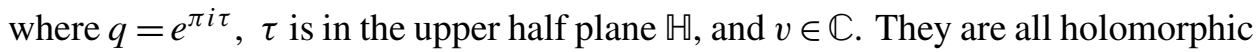
functions for $(v, \tau) \in \mathbb{C} \times \mathbb{W}$. Let $\theta^{\prime}(0, \tau)=\left.(\partial / \partial v) \theta(v, \tau)\right|_{v=0}$. The Jacobi theta 
functions satisfy the following relations [Chandrasekharan 1985]:

$$
\begin{aligned}
\theta(v+1, \tau) & =-\theta(v, \tau), & \theta(v+\tau, \tau) & =-\frac{1}{q} e^{-2 \pi i v} \theta(v, \tau), \\
\theta_{1}(v+1, \tau) & =-\theta_{1}(v, \tau), & \theta_{1}(v+\tau, \tau) & =\frac{1}{q} e^{-2 \pi i v} \theta_{1}(v, \tau), \\
\theta_{2}(v+1, \tau) & =\theta_{2}(v, \tau), & \theta_{2}(v+\tau, \tau) & =-\frac{1}{q} e^{-2 \pi i v} \theta_{2}(v, \tau), \\
\theta_{3}(v+1, \tau) & =\theta_{3}(v, \tau), & \theta_{3}(v+\tau, \tau) & =\frac{1}{q} e^{-2 \pi i v} \theta_{3}(v, \tau) .
\end{aligned}
$$

Therefore it's not hard to deduce how the theta functions vary along the lattice $\Gamma=\{m+n \tau \mid m, n \in \mathbb{Z}\}$. We have

$$
\theta(v+m, \tau)=(-1)^{m} \theta(v, \tau)
$$

and

$$
\begin{aligned}
\theta(v+ & n \tau, \tau)=-\frac{1}{q} e^{-2 \pi i(v+(n-1) \tau)} \theta(v+(n-1) \tau, \tau) \\
& =-\frac{1}{q} e^{-2 \pi i(v+(n-1) \tau)}\left(-\frac{1}{q}\right) e^{-2 \pi i(v+(n-2) \tau)} \theta(v+(n-2) \tau, \tau) \\
& =(-1)^{n} \frac{1}{q^{n}} e^{-2 \pi i[(v+(n-1) \tau)+(v+(n-2) \tau)+\cdots+v]} \theta(v, \tau) \\
& =(-1)^{n} \frac{1}{q^{n}} e^{-2 \pi i n v-\pi i n(n-1) \tau} \theta(v, \tau) \\
& =(-1)^{n} e^{-2 \pi i n v-\pi i n^{2} \tau} \theta(v, \tau) .
\end{aligned}
$$

Similarly, we have

$$
\begin{array}{ll}
\theta_{1}(v+m, \tau)=(-1)^{m} \theta_{1}(v, \tau), & \theta_{1}(v+n \tau, \tau)=e^{-2 \pi i n v-\pi i n^{2} \tau} \theta_{1}(v, \tau) ; \\
\theta_{2}(v+m, \tau)=\theta_{2}(v, \tau), & \theta_{2}(v+n \tau, \tau)=(-1)^{n} e^{-2 \pi i n v-\pi i n^{2} \tau} \theta_{2}(v, \tau) ; \\
\theta_{3}(v+m, \tau)=\theta_{3}(v, \tau), & \theta_{3}(v+n \tau, \tau)=e^{-2 \pi i n v-\pi i n^{2} \tau} \theta_{3}(v, \tau) .
\end{array}
$$

\section{Proof of Theorem 1.1}

Let $i: V_{\left(d_{p q}\right)} \rightarrow \mathbb{C P}^{n_{1}} \times \mathbb{C P}^{n_{2}} \times \cdots \times \mathbb{C P}^{n_{s}}$ be the inclusion. It's not hard to see that

$$
i^{*} T_{\mathbb{R}}\left(\mathbb{C P}^{n_{1}} \times \mathbb{C P}^{n_{2}} \times \cdots \times \mathbb{C P}^{n_{s}}\right) \cong T V_{\left(d_{p q}\right)} \bigoplus i^{*}\left(\bigoplus_{p=1}^{t}\left(\bigotimes_{q=1}^{s} P_{q}^{*} \mathbb{O}\left(d_{p q}\right)\right)\right),
$$


where we forget the complex structure of the line bundles $\bigotimes_{q=1}^{s} P_{q}^{*} \mathcal{O}\left(d_{p q}\right)$ for $1 \leq p \leq t$. Therefore for the total Stiefel-Whitney class, we have

$$
i^{*} w\left(T_{\mathbb{R}}\left(\mathbb{C P}^{n_{1}} \times \mathbb{C P}^{n_{2}} \times \cdots \times \mathbb{C} \mathrm{P}^{n_{s}}\right)\right)=w\left(T V_{\left(d_{p q}\right)}\right) \prod_{p=1}^{t} i^{*} w\left(\bigotimes_{q=1}^{s} P_{q}^{*} \mathbb{O}\left(d_{p q}\right)\right),
$$

or more precisely

$$
i^{*}\left(\prod_{q=1}^{s}\left(1+x_{q}\right)^{n_{q}+1}\right) \equiv w\left(T V_{\left(d_{p q}\right)}\right) \prod_{p=1}^{t} i^{*}\left(1+\sum_{q=1}^{s} d_{p q} x_{q}\right) \quad(\bmod 2) .
$$

By (3-7), we can easily see that

$$
w_{1}\left(T V_{\left(d_{p q}\right)}\right)=0 \quad \text { and } \quad w_{2}\left(T V_{\left(d_{p q}\right)}\right) \equiv \sum_{q=1}^{s}\left(n_{q}+1-\sum_{p=1}^{t} d_{p q}\right) i^{*} x_{q} \quad(\bmod 2) .
$$

As for the total rational Pontryagin class, we have

$$
i^{*} p\left(T_{\mathbb{R}}\left(\mathbb{C P}^{n_{1}} \times \mathbb{C} \mathrm{P}^{n_{2}} \times \cdots \times \mathbb{C P}^{n_{s}}\right)\right)=p\left(T V_{\left(d_{p q}\right)}\right) \prod_{p=1}^{t} i^{*} p\left(\bigotimes_{q=1}^{s} P_{q}^{*} \mathcal{O}\left(d_{p q}\right)\right),
$$

or

$$
p\left(V_{\left(d_{p q}\right)}\right)=\prod_{q=1}^{s}\left(1+\left(i^{*} x_{q}\right)^{2}\right)^{n_{q}+1} \prod_{p=1}^{t}\left(1+\left(\sum_{q=1}^{s} d_{p q} i^{*} x_{q}\right)^{2}\right)^{-1} .
$$

Hence we have

$$
\begin{aligned}
p_{1}\left(V_{\left(d_{p q}\right)}\right) & =\sum_{q=1}^{s}\left(n_{q}+1\right)\left(i^{*} x_{q}\right)^{2}-\sum_{p=1}^{t}\left(\sum_{q=1}^{s} d_{p q} i^{*} x_{q}\right)^{2} \\
& =\sum_{q=1}^{s}\left(n_{q}+1-\sum_{p=1}^{t} d_{p q}^{2}\right)\left(i^{*} x_{q}\right)^{2}-\sum_{\substack{1 \leq u, v \leq s \\
u \neq v}}\left(\sum_{p=1}^{t} d_{p u} d_{p v} i^{*} x_{u} i^{*} x_{v}\right) .
\end{aligned}
$$

Let $i_{!}: H^{*}\left(V_{\left(d_{p q}\right)}, \mathbb{Q}\right) \rightarrow H^{*+2 t}\left(\mathbb{C P}^{n_{1}} \times \mathbb{C P}^{n_{2}} \times \cdots \times \mathbb{C P}^{n_{s}}, \mathbb{Q}\right)$ be the push forward. Thus if $p_{1}\left(V_{\left(d_{p q}\right)}\right)=0$, then

$$
i ! p_{1}\left(V_{\left(d_{p q}\right)}\right)=i ! i^{*}\left(\sum_{q=1}^{s}\left(n_{q}+1-\sum_{p=1}^{t} d_{p q}^{2}\right) x_{q}^{2}-\sum_{\substack{1 \leq u, v \leq s \\ u \neq v}}\left(\sum_{p=1}^{t} d_{p u} d_{p v} x_{u} x_{v}\right)\right)=0
$$


that is,

$$
\left(\prod_{p=1}^{t}\left(\sum_{q=1}^{s} d_{p q} x_{q}\right)\right)\left(\sum_{q=1}^{s}\left(n_{q}+1-\sum_{p=1}^{t} d_{p q}^{2}\right) x_{q}^{2}-\sum_{\substack{1 \leq u, v \leq s \\ u \neq v}}\left(\sum_{p=1}^{t} d_{p u} d_{p v} x_{u} x_{v}\right)\right)=0
$$

in $H^{2 t+4}\left(\mathbb{C P}^{n_{1}} \times \mathbb{C P}^{n_{2}} \times \cdots \times \mathbb{C P}^{n_{s}}, \mathbb{Q}\right)$. If $m_{q}+2 \leq n_{q}$ for $1 \leq q \leq s$, then the left hand side of the above equality should not only be a zero element in the cohomology ring but should also be a zero polynomial. Note that the polynomial ring is an integral domain. Therefore at least one of its factors should be zero. But $\prod_{p=1}^{t}\left(\sum_{q=1}^{s} d_{p q} x_{q}\right)$ is nonzero. This means

$$
\sum_{q=1}^{s}\left(n_{q}+1-\sum_{p=1}^{t} d_{p q}^{2}\right) x_{q}^{2}-\sum_{\substack{1 \leq u, v \leq s \\ u \neq v}}\left(\sum_{p=1}^{t} d_{p u} d_{p v} x_{u} x_{v}\right)=0,
$$

and consequently these identities hold:

$$
\begin{aligned}
n_{q}+1-\sum_{p=1}^{t} d_{p q}^{2}=0 & \text { for } 1 \leq q \leq s, \\
\sum_{p=1}^{t} d_{p u} d_{p v} & =0 \quad \text { for } 1 \leq u, v \leq s \text { and } u \neq v .
\end{aligned}
$$

Note that $n_{q}+1-\sum_{p=1}^{t} d_{p q}^{2} \equiv n_{q}+1-\sum_{p=1}^{t} d_{p q}(\bmod 2)$ for $1 \leq q \leq s$. Hence the first of (3-8) implies that $w_{2}\left(T V_{\left(d_{p q}\right)}\right)=0$.

In summary:

Proposition 3.1. If $m_{q}+2 \leq n_{q}$ for $1 \leq q \leq s$, then $p_{1}\left(V_{\left(d_{p q}\right)}\right)=0$ implies $V_{\left(d_{p q}\right)}$ is spin. Therefore if $m_{q}+2 \leq n_{q}$ for $1 \leq q \leq s$, then $V_{\left(d_{p q}\right)}$ is string if and only if any of the following holds:

(1) $p_{1}\left(V_{\left(d_{p q}\right)}\right)=0$.

(2) Equation (3-8) is satisfied.

(3) The matrix $D$ defined in (1-2) satisfies $\left\|\operatorname{col}_{\mathrm{q}} \mathrm{D}\right\|^{2}=n_{q}+1$ for $1 \leq q \leq s$, and any two of its columns are orthogonal, that is,

$$
D^{t} D=\operatorname{diag}\left(n_{1}+1, \ldots, n_{s}+1\right) .
$$

Proof of Theorem 1.1. Denote the fundamental class of $V_{\left(d_{p q}\right)}$ in $H_{4 k}\left(V_{\left(d_{p q}\right)}, \mathbb{Z}\right)$ by $\left[V_{\left(d_{p q}\right)}\right]$. Then according to (1-1) and the multiplicative property of the Witten 
genus, up to a constant scalar,

$$
\begin{aligned}
& \varphi_{W}\left(V_{\left(d_{p q}\right)}\right)=\left(\left(\prod_{q=1}^{s} \frac{\left(i^{*} x_{q}\right)^{n_{q}+1}}{\left(\frac{\theta\left(i^{*} x_{q}, \tau\right)}{\theta^{\prime}(0, \tau)}\right)^{n_{q}+1}}\right)\left(\prod_{p=1}^{t} \frac{\left(\sum_{q=1}^{s} d_{p q} i^{*} x_{q}\right)^{-1}}{\left(\frac{\theta\left(\sum_{q=1}^{s} d_{p q} i^{*} x_{q}, \tau\right)}{\theta^{\prime}(0, \tau)}\right)}\right)\right)\left[V_{\left(d_{p q}\right)}\right] \\
& =\left(\left(\prod_{q=1}^{s} \frac{x_{q}^{n_{q}+1}}{\left(\frac{\theta\left(x_{q}, \tau\right)}{\theta^{\prime}(0, \tau)}\right)^{n_{q}+1}}\right)\left(\prod_{p=1}^{t} \frac{1}{\left(\frac{\theta\left(\sum_{q=1}^{s} d_{p q} x_{q}, \tau\right)}{\theta^{\prime}(0, \tau)}\right)}\right)\left[\mathbb{C} \mathrm{P}^{n_{1}} \times \mathbb{C} \mathrm{P}^{n_{2}} \times \cdots \times \mathbb{C} \mathrm{P}^{n_{s}}\right]\right. \\
& =\operatorname{coefficient} \text { of } x_{1}^{n_{1}} \cdots x_{s}^{n_{s}} \text { in } \frac{\left(\prod_{q=1}^{s} x_{q}^{n_{q}+1}\right)\left(\prod_{p=1}^{t} \frac{\theta\left(\sum_{q=1}^{s} d_{p q} x_{q}, \tau\right)}{\theta^{\prime}(0, \tau)}\right)}{\prod_{q=1}^{s}\left(\frac{\theta\left(x_{q}, \tau\right)}{\theta^{\prime}(0, \tau)}\right)^{n_{q}+1}} \\
& =\operatorname{Res}_{0}\left(\frac{\left(\prod_{p=1}^{t} \frac{\theta\left(\sum_{q=1}^{s} d_{p q} x_{q}, \tau\right)}{\theta^{\prime}(0, \tau)}\right) d x_{1} \wedge \cdots \wedge d x_{s}}{\prod_{q=1}^{s}\left(\frac{\theta\left(x_{q}, \tau\right)}{\theta^{\prime}(0, \tau)}\right)^{n_{q}+1}}\right) .
\end{aligned}
$$

The second equality above follows from Poincare duality. Now, set

$$
g\left(x_{1}, \ldots, x_{s}\right)=\prod_{p=1}^{t} \frac{\theta\left(\sum_{q=1}^{s} d_{p q} x_{q}, \tau\right)}{\theta^{\prime}(0, \tau)} \quad \text { and } \quad f_{q}\left(x_{q}\right)=\left(\frac{\theta\left(x_{q}, \tau\right)}{\theta^{\prime}(0, \tau)}\right)^{n_{q}+1}
$$

for $1 \leq q \leq s$, and define

$$
\omega=\frac{g\left(x_{1}, \ldots, x_{s}\right) d x_{1} \wedge \cdots \wedge d x_{s}}{f_{1}\left(x_{1}\right) \cdots f_{s}\left(x_{s}\right)} .
$$

Then up to a constant scalar,

$$
\varphi_{W}\left(V_{\left(d_{p q}\right)}\right)=\operatorname{Res}_{(0,0, \ldots, 0)} \omega .
$$

By (2-5),

$$
\begin{aligned}
g\left(x_{1}+1, x_{2}, \ldots, x_{s}\right) & =\prod_{p=1}^{t} \frac{\theta\left(\sum_{q=1}^{s} d_{p q} x_{q}+d_{p 1}, \tau\right)}{\theta^{\prime}(0, \tau)} \\
& =(-1)^{d_{11}+\cdots+d_{t 1}} \prod_{p=1}^{t} \frac{\theta\left(\sum_{q=1}^{s} d_{p q} x_{q}, \tau\right)}{\theta^{\prime}(0, \tau)}
\end{aligned}
$$

and

$$
f_{1}\left(x_{1}+1\right)=\left(\frac{\theta\left(x_{1}+1, \tau\right)}{\theta^{\prime}(0, \tau)}\right)^{n_{1}+1}=(-1)^{n_{1}+1}\left(\frac{\theta\left(x_{1}, \tau\right)}{\theta^{\prime}(0, \tau)}\right)^{n_{1}+1} .
$$

Thus

$$
\frac{g\left(x_{1}+1, \ldots, x_{s}\right)}{f_{1}\left(x_{1}+1\right) \cdots f_{s}\left(x_{s}\right)}=(-1)^{\left(d_{11}+\cdots+d_{t 1}\right)-\left(n_{1}+1\right)} \frac{g\left(x_{1}, \ldots, x_{s}\right)}{f_{1}\left(x_{1}\right) \cdots f_{s}\left(x_{s}\right)}
$$


By (3-8), $\left(d_{11}+\cdots+d_{t 1}\right)-\left(n_{1}+1\right) \equiv\left(d_{11}^{2}+\cdots+d_{t 1}^{2}\right)-\left(n_{1}+1\right)=0(\bmod 2)$. Thus one obtains that

$$
\frac{g\left(x_{1}+1, \ldots, x_{s}\right)}{f_{1}\left(x_{1}+1\right) \cdots f_{s}\left(x_{s}\right)}=\frac{g\left(x_{1}, \ldots, x_{s}\right)}{f_{1}\left(x_{1}\right) \cdots f_{s}\left(x_{s}\right)} .
$$

Similarly, we have

$$
\frac{g\left(x_{1}, \ldots, x_{q}+1, \ldots, x_{s}\right)}{f_{1}\left(x_{1}\right) \cdots f_{q}\left(x_{q}+1\right) \cdots f_{s}\left(x_{s}\right)}=\frac{g\left(x_{1}, \ldots, x_{s}\right)}{f_{1}\left(x_{1}\right) \cdots f_{s}\left(x_{s}\right)} \quad \text { for } 1 \leq q \leq s .
$$

On the other hand, by (2-5) and (2-6),

$$
\begin{aligned}
g\left(x_{1}+\tau, x_{2}, \ldots, x_{s}\right)=\prod_{p=1}^{t} \frac{\theta\left(\sum_{q=1}^{s} d_{p q} x_{q}+d_{p 1} \tau, \tau\right)}{\theta^{\prime}(0, \tau)} \\
=\prod_{p=1}^{t}(-1)^{d_{p 1}} e^{-2 \pi i d_{p 1}\left(\sum_{q=1}^{s} d_{p q} x_{q}\right)-\pi i d_{p 1}^{2} \tau} \cdot \frac{\theta\left(\sum_{q=1}^{s} d_{p q} x_{q}, \tau\right)}{\theta^{\prime}(0, \tau)} \\
=(-1)^{d_{11}+\cdots+d_{t 1}} e^{-2 \pi i \sum_{p=1}^{t} d_{p 1}\left(\sum_{q=1}^{s} d_{p q} x_{q}\right)-\pi i \tau\left(\sum_{p=1}^{t} d_{p 1}^{2}\right)} \cdot \frac{\theta\left(\sum_{q=1}^{s} d_{p q} x_{q}, \tau\right)}{\theta^{\prime}(0, \tau)} \\
=(-1)^{d_{11}+\cdots+d_{t 1}} e^{-2 \pi i \sum_{p=1}^{t} d_{p 1}\left(\sum_{q=1}^{s} d_{p q} x_{q}\right)-\pi i \tau\left(\sum_{p=1}^{t} d_{p 1}^{2}\right)} g\left(x_{1}, x_{2}, \ldots, x_{s}\right)
\end{aligned}
$$

and

$$
\begin{aligned}
f_{1}\left(x_{1}+\tau\right) & =\left(\frac{\theta\left(x_{1}+\tau, \tau\right)}{\theta^{\prime}(0, \tau)}\right)^{n_{1}+1} \\
& =\left(-e^{-2 \pi i x_{1}-\pi i \tau} \frac{\theta\left(x_{1}, \tau\right)}{\theta^{\prime}(0, \tau)}\right)^{n_{1}+1} \\
& =(-1)^{n_{1}+1} e^{-2 \pi i\left(n_{1}+1\right) x_{1}-\pi i \tau\left(n_{1}+1\right)}\left(\frac{\theta\left(x_{1}, \tau\right)}{\theta^{\prime}(0, \tau)}\right)^{n_{1}+1} \\
& =(-1)^{n_{1}+1} e^{-2 \pi i\left(n_{1}+1\right) x_{1}-\pi i \tau\left(n_{1}+1\right)} f_{1}\left(x_{1}\right) .
\end{aligned}
$$

Therefore

(3-11) $\frac{g\left(x_{1}+\tau, \ldots, x_{s}\right)}{f_{1}\left(x_{1}+\tau\right) \cdots f_{s}\left(x_{s}\right)}=(-1)^{d_{11}+\cdots+d_{t 1}-n_{1}-1} \frac{g\left(x_{1}, \ldots, x_{s}\right)}{f_{1}\left(x_{1}\right) \cdots f_{s}\left(x_{s}\right)}$

$\times \exp \left(-2 \pi i \sum_{p=1}^{t} d_{p 1}\left(\sum_{q=1}^{s} d_{p q} x_{q}\right)-\pi i \tau\left(\sum_{p=1}^{t} d_{p 1}^{2}\right)+2 \pi i\left(n_{1}+1\right) x_{1}+\pi i \tau\left(n_{1}+1\right)\right)$.

However, $d_{11}+\cdots+d_{t 1}-n_{1}-1 \equiv d_{11}^{2}+\cdots+d_{t 1}^{2}-n_{1}-1(\bmod 2)$, and the argument of exp in (3-11) is equal to

$$
\pi i \tau\left(\left(n_{1}+1\right)-\sum_{p=1}^{t} d_{p 1}^{2}\right)+2 \pi i\left(\left(n_{1}+1\right)-\sum_{p=1}^{t} d_{p 1}^{2}\right) x_{1}-2 \pi i \sum_{q=2}^{s}\left(\sum_{p=1}^{t} d_{p 1} d_{p q} x_{q}\right) .
$$


Therefore by (3-8), we have $d_{11}+\cdots+d_{t 1}-n_{1}-1 \equiv 0(\bmod 2)$, and

$$
-2 \pi i \sum_{p=1}^{t} d_{p 1}\left(\sum_{q=1}^{s} d_{p q} x_{q}\right)-\pi i \tau\left(\sum_{p=1}^{t} d_{p 1}^{2}\right)+2 \pi i\left(n_{1}+1\right) x_{1}+\pi i \tau\left(n_{1}+1\right)=0 .
$$

Consequently, by (3-11), we obtain that

$$
\frac{g\left(x_{1}+\tau, \ldots, x_{s}\right)}{f_{1}\left(x_{1}+\tau\right) \cdots f_{s}\left(x_{s}\right)}=\frac{g\left(x_{1}, \ldots, x_{s}\right)}{f_{1}\left(x_{1}\right) \cdots f_{s}\left(x_{s}\right)} .
$$

Similarly, one also obtains that

$$
\frac{g\left(x_{1}, \ldots, x_{q}+\tau, \ldots, x_{s}\right)}{f_{1}\left(x_{1}\right) \cdots f_{q}\left(x_{q}+\tau\right) \cdots f_{s}\left(x_{s}\right)}=\frac{g\left(x_{1}, \ldots, x_{s}\right)}{f_{1}\left(x_{1}\right) \cdots f_{s}\left(x_{s}\right)} \quad \text { for } 1 \leq q \leq s .
$$

Therefore from (3-10) and (3-12), we see that $\omega$ can be viewed as a meromorphic $s$-form defined on the product $(\mathbb{C} / \Gamma)^{s}$, an $s$-tori which is a compact complex manifold.

$\theta(v, \tau)$ has the lattice points $m+n \tau, m, n \in \mathbb{Z}$ as its simple zero points [Chandrasekharan 1985]. We therefore see that $\omega$ has pole divisors

$$
\{0\} \times(\mathbb{C} / \Gamma)^{s-1},(\mathbb{C} / \Gamma) \times\{0\} \times(\mathbb{C} / \Gamma)^{s-2}, \ldots,(\mathbb{C} / \Gamma)^{s-1} \times\{0\} .
$$

So $(0,0, \ldots, 0)$ is the unique intersection point of these polar divisors.

Therefore by the residue theorem on compact complex manifolds, we directly deduce $\operatorname{Res}_{(0,0, \ldots, 0)} \omega=0$. By (3-9), we get $\varphi_{W}\left(V_{\left(d_{p q}\right)}\right)=\operatorname{Res}_{(0,0, \ldots, 0)} \omega=0$.

\section{Acknowledgement}

F. Han is grateful to Professor Peter Teichner for a lot of discussion and help. He also thanks Professor Kefeng Liu for inspiring suggestions. Q. Chen is grateful to Professor Nicolai Reshetikhin for his interest and support. Thanks also go to Professors Friedrich Hirzebruch, Stephan Stolz, and Weiping Zhang for their interest and many discussions with us. We would like to thank Professors Michael Joachim and Serge Ochanine for inspiring communications with us. The paper was finished when the second author was visiting the Max-Planck-Institut für Mathematik at Bonn.

\section{References}

[Alvarez-Gaumé and Witten 1984] L. Alvarez-Gaumé and E. Witten, "Gravitational anomalies", Nuclear Phys. B 234:2 (1984), 269-330. MR 85j:81062

[Atiyah 1967] M. F. Atiyah, K-theory, Lecture notes by D. W. Anderson, W. A. Benjamin, New York-Amsterdam, 1967. MR 36 \#7130 Zbl 0159.53302

[Chandrasekharan 1985] K. Chandrasekharan, Elliptic functions, Grundlehren der Mathematischen Wissenschaften 281, Springer, Berlin, 1985. MR 87e:11058 Zbl 0575.33001 
[Chen and Han 2006] Q. Chen and F. Han, "Modular invariance and twisted anomaly cancellations for characteristic numbers", preprint, 2006. Accepted for publication in Trans. Amer. Math. Soc. arXiv math.DG/0605415

[Gorbounov and Malikov 2004] V. Gorbounov and F. Malikov, "Vertex algebras and the LandauGinzburg/Calabi-Yau correspondence”, Mosc. Math. J. 4:3 (2004), 729-779, 784. MR 2005m: 17037 Zbl 1079.14050

[Gorbounov and Ochanine 2006] V. Gorbounov and S. Ochanine, "Mirror symmetry formulae for the elliptic genus of complete intersections", preprint, 2006. Accepted for publication in Topology. arXiv math.AT/0603196

[Griffiths and Harris 1994] P. Griffiths and J. Harris, Principles of algebraic geometry, Wiley Classics Library, John Wiley \& Sons, New York, 1994. MR 95d:14001 Zbl 0836.14001

[Hirzebruch et al. 1992] F. Hirzebruch, T. Berger, and R. Jung, Manifolds and modular forms, Aspects of Mathematics, E20, Friedr. Vieweg \& Sohn, Braunschweig, 1992. MR 94d:57001 Zbl 0767.57014

[Liu 1995a] K. Liu, "Modular invariance and characteristic numbers", Comm. Math. Phys. 174:1 (1995), 29-42. MR 96m:57034 Zbl 0867.57021

[Liu 1995b] K. Liu, "On modular invariance and rigidity theorems", J. Differential Geom. 41:2 (1995), 343-396. MR 96f:58152 Zbl 0836.57024

[Liu 1996] K. Liu, “On elliptic genera and theta-functions”, Topology 35:3 (1996), 617-640. MR 97h:58149 Zbl 0858.57034

[Ochanine 1981] S. Ochanine, "Signature modulo 16, invariants de Kervaire généralisés et nombres caractéristiques dans la K-théorie réelle”, Mém. Soc. Math. France (N.S.) 5 (1981), 142. MR 83j:57014 Zbl 0462.57012

[Stolz 1996] S. Stolz, "A conjecture concerning positive Ricci curvature and the Witten genus", Math. Ann. 304:4 (1996), 785-800. MR 96k:58209 Zbl 0856.53033

[Witten 1988] E. Witten, "The index of the Dirac operator in loop space”, pp. 161-181 in Elliptic curves and modular forms in algebraic topology (Princeton, NJ, 1986), edited by P. S. Landweber, Lecture Notes in Math. 1326, Springer, Berlin, 1988. MR 970288 Zbl 0679.58045

[Zhang 1992] W. Zhang, "Elliptic genera and Rokhlin congruences", preprint IHES/M/92/76, 1992.

[Zhang 2001] W. Zhang, Lectures on Chern-Weil theory and Witten deformations, Nankai Tracts in Mathematics 4, World Scientific, River Edge, NJ, 2001. MR 2002m:58032 Zbl 0993.58014

Received January 18, 2007.

\section{Qingtao Chen}

DEPARTMENT OF MATHEMATICS

UNIVERSITY OF CALIFORNIA

BERKELEY, CA 94720-3840

UNITED STATES

chenqtao@math.berkeley.edu

FEI HAN

DEPARTMENT OF MATHEMATICS

UNIVERSITY OF CALIFORNIA

BERKELEY, CA 94720-3840

UNITED STATES

feihan@math.berkeley.edu 